\title{
Evaluación de Diferentes Formas de Deseo Erótico y su Regulación: Cuestionario Individual de Deseo Erótico y Regulación - CIDER
}

\author{
Evaluation of Different Forms of Erotic Desire and their Regulation: Individual \\ Questionnaire of Erotic Desire and Regulation - CIDER
}

\author{
Lara Salguero Lucas ${ }^{1}$, Miguel Ángel Pérez Nieto ${ }^{2}$ y Silberio Sáez Sesma ${ }^{3}$
}

\begin{abstract}
Resumen
El objetivo fue desarrollar una escala de autorregistro del nivel y la regulación del deseo erótico de hombres y mujeres con pareja. Método: se contó con una muestra de 279 participantes con la que se llevó a cabo un análisis factorial exploratorio. Resultados: el AFE dio lugar a una escala de 10 ítems formada por tres componentes. Un primer factor que hace referencia al deseo erótico fuera de la pareja, un segundo factor que hace referencia a la regulación del deseo erótico y un tercer factor referido al deseo diádico hacia la pareja. La consistencia interna muestra niveles aceptables para cada factor. Siendo Alfa de Cronbach .79 para el primer factor, .69 para el segundo factor y .67 para el tercer factor. Conclusiones: los resultados revelan la necesidad de entender y medir el deseo erótico en pareja como un constructo diferente así como entender el componente motivacional del deseo con la finalidad de trabajar en su regulación.
\end{abstract}

Palabras clave: análisis factorial, deseo erótico, escala de medición, regulación

\begin{abstract}
The objective was to develop a self-registration scale of the level and regulation of the erotic desire of males and females with a partner. Method: a sample of 279 subjects was used on which an exploratory factor analysis was conducted. Results: the EFA resulted in a 10-item scale consisting of three components. A first factor referring to erotic desire outside the couple, a second factor referring to the regulation of erotic desire and a third factor referring to the dyadic desire towards the couple. Internal consistency showed acceptable levels for each factor. Cronbach's Alpha was .79 for the first factor, .69 for the second factor and .67 for the third factor. Conclusions: the results reveal the need to understand and measure erotic desire as a couple as a different construct as well as to understand the motivational component of desire in order to work on its regulation.
\end{abstract}

Keywords: erotic desire, factor analysis, measurement scale, regulation

\footnotetext{
${ }^{1}$ Doctoranda en Psicologia y Sexóloga. Profesora UCJC, Universidad Camilo José Cela, Facultad de Ciencias de la Salud. C/ Castillo de Alarcón, 49 - Urb. Villafranca del Castillo - 28692 Madrid, España. Correo: lara.salguero@ucjc.edu

${ }^{2}$ Doctor en Psicologia. Decano de la Facultad de Salud y Profesor en UCJC, Universidad Camilo José Cela. C/ Castillo de Alarcón, 49 Urb. Villafranca del Castillo- 28692 Madrid, España. Correo: mperez@ucjc.edu

${ }^{3}$ Doctor en Psicologia. Director Instituro Amaltea. Instituto de Sexología y Psicoterapia AMALTEA, 50007 Zaragoza, España. Correo: silberio@amaltea.org

Revista Iberoamericana de Diagnóstico y Evaluación - e Avaliação Psicológica. RIDEP · No61 · Vol.4 · 177-188 · 2021 ISSN: 1135-3848 print /2183-6051online
} 


\section{Introducción}

La medición del deseo erótico a través de pruebas de autorregistro es a día de hoy la forma más habitual por los profesionales para valorar los niveles de deseo erótico. A lo largo de los años se han desarrollado instrumentos (e.g: Derogatis \& Melisaratos, 1979; Master et al., 1994; Spector et al., 1996) basados en teorías acerca del origen y el funcionamiento del deseo que han ayudado en la investigación y el trabajo clínico de los expertos.

Es pertinente revisar el origen del estudio del deseo erótico para posteriormente revisar los instrumentos de medida más utilizados.

Uno de los primeros autores que introdujo desde la psicología el constructo de deseo erótico fue Sigmund Freud quien en 1905 habló de la existencia de una necesidad sexual en el hombre definiéndola como pulsión sexual con el objetivo de la unión sexual entre dos sexos. Entendiendo así desde las teorías psicoanalíticas de la época el deseo sexual como una libido irrefrenable expresada en conductas sexuales. A diferencia de estas teorías donde los estímulos internos tenían un papel principal en el desarrollo de las pulsiones sexuales, autores como Beach (1956), desde las teorías del aprendizaje, dieron más importancia a factores externos y ambientales en la aparición de intereses sexuales. $\mathrm{Si}$ revisamos estas $\mathrm{y}$ posteriores aportaciones de los diferentes autores en su intento por explicar el deseo erótico, se destaca la dificultad que existe en definirlo de forma clara y limitada. Una de las principales causas de esta dificultad se debe, como ya afirmaron Rosen y Leiblum (1987), al desarrollo de enfoques terapéuticos en ausencia de modelos teóricos de deseo erótico. Como comenta Gómez Zapiain (1995), la estructura del deseo erótico tiene un origen biofisiológico con una meta reproductiva siendo ésta trascendida por el ser humano y convirtiéndose dicho constructo en una de las mayores motivaciones de la existencia. Desde que por primera vez Kaplan (1979) hablara del Deseo Sexual como una fase del modelo de respuesta sexual humana en sus trabajos e investigaciones, muchos han sido los autores que han querido saber más acerca de lo que hoy se conoce en sexología como deseo erótico. Según Kaplan (1982) el deseo sexual es un impulso que se deriva de una activación de un sistema neuronal específico, derivándose después las fases de excitación y orgasmo que afectan directamente a los genitales. Como se entiende desde la teoría de la autora, el deseo sexual sería la primera fase de la respuesta sexual humana seguida de la respuesta genital. Más adelante, a finales de los años 80, Leiblum y Rosen (1987) postulan que el deseo sexual sería el resultado de una combinación de dos factores principales; una estimulación sexual y un correcto funcionamiento neuroendocrino. Entendiendo la importancia de la biología y el ambiente en el funcionamiento del deseo erótico, autores como Gil-Verona (2009) ya afirmaron que el deseo tiene como características principales la finalidad de satisfacer la necesidad del sujeto y además, éste va siempre acompañado de emociones. Con relación a esta teoría del deseo erótico y resaltando el componente emocional, en la actualidad se acepta el triple plano en lo relativo al funcionamiento de este constructo.

Haciendo una breve revisión de las teorías más importantes que han explicado el deseo en relación con tres componentes principales, Marañon (1925) ya postuló la idea de un plano vegetativo, psíquico y expresivo en lo relativo al deseo. Más adelante, y entendida como una de las teorías principales de la sexología en relación al deseo erótico, Levine (1984) plantea una interesante conceptualización proponiendo tres componentes principales en la definición de dicho constructo; un componente biológico, un componente motivacional y un componente cognitivo concluyendo que el deseo sexual puede entenderse como un indicador de la capacidad que tiene un sujeto de integrar el aspecto sexual biológico, intrapsíquico e interpersonal, siendo este por tanto, una capacidad integradora de la personalidad. Por su parte, Fuertes y López (1997) conceptualizan el deseo también desde la interacción de tres componentes fundamentales; una base neurofisiológica, una disposición emocional y cognitiva positiva y la presencia de inductores eficaces de sensaciones sexuales. Más recientemente y con relación al deseo erótico femenino, Basson (2000) quien afirmó la necesidad de entender un modelo de respuesta sexual femenino diferente al de los hombres, resaltó la importancia de la motivación en la activación del deseo erótico de las mujeres y entendió que el deseo podría comenzar después de 
una excitación sexual y no desde el primer momento y de forma espontánea como se podría plantear en un modelo masculino. Tras esta revisión se entiende que la dificultad en definir un constructo tan complejo dificulta a su vez su posible evaluación por parte de los profesionales.

A lo largo de los años varios han sido los autores que a través de diferentes teorías y postulados acerca de qué es y cómo se comporta el deseo erótico, han diseñado instrumentos de evaluación con la intención de poder concluir una medida clara y concisa que ayude a su interpretación y posterior intervención. Uno de los problemas a los que se enfrenta la medición de este constructo es la asimilación por parte de los investigadores de entender la conducta y/o el comportamiento sexual como sinónimo de deseo erótico. En esta linea, y llevando a cabo un estudio acerca de la experiencia de deseo erótico en alumnos universitario, (Beck et al., 1991) concluyeron que el deseo erótico y el comportamiento sexual no tienen por qué ser constructos semejantes, puesto que en ocasiones se pueden dar comportamientos sexuales sin que haya deseo erótico previo.

Con todo ello, sería un error el desarrollo de instrumentos de medida que pretendan medir la cantidad de deseo erótico de un sujeto a través de la cantidad de conductas sexuales que tenga. Esto se relaciona con la idea de Nuttin (1982) quien afirma que entender una conducta únicamente en términos motivacionales es hacer un entendimiento parcial de la misma, ya que existen otras variables, personales, fisiólogicas o situacionales que pueden influir en el desarrollo de la conducta.

Entendiendo el deseo como proceso motivacional no sería correcto derivar entonces que dichas conductas sexuales se deben únicamente a la motivación. Pues como ya afirmaron Basson et al. (2003), en relación al deseo femenino, existen múltiples motivos por los cuales un sujeto puede iniciar un comportamiento erótico no siendo éstos únicamente, el anhelo de experimentar placer.

Uno de los instrumentos presentes en la literatura es el The Derogatis Sexual Functioning Inventory (DSFI) el cual fue diseñado como prueba genérica del funcionamiento sexual, muestreo y medición de los componentes principales de dicho funcionamiento. (Derogatis \& Melisaratos, 1979). Más tarde, Kaplan y Harder (1991) diseñaron la Escala de Conflicto Sexual para mujeres con la idea de poder medir el grado en el que las mujeres podían experimentar molestias psicológicas y/o emocionales en relación a su deseo erótico y su excitación (Kaplan \& Harder, 1991). Esta escala no es una medición del deseo erótico y aunque mide aspectos que sí pueden influir en el desarrollo del deseo, no mide como tal dicho constructo. Más tarde, en 1994, Máster, Johnson y Kolodny presentan el Test de Deseo Sexual Inhibido con la intención de estudiar y poder cuantificar dificultades del deseo sexual hipoactivo. El instrumento hace referencia a conductas sexuales de origen genital y coital y basa la falta de deseo erótico únicamente en falta de ganas por mantener conductas sexuales genitales. Por su parte, Spector y colaboradores a finales de los años 90 diseñaron el Sexual Desire Inventory el cual parte de la idea de deseo erótico como el interés de una persona por comportarse de forma sexual (Spector et el., 1996). El inventario consta de 13 ítems los cuales se dividen en dos factores; uno de ellos centrado en el deseo diádico y el otro en el deseo solitario. $\mathrm{Si}$ bien es cierto que el inventario se centra en medir el componente cognitivo no limitándose únicamente al componente conductual, este sigue siendo un instrumento limitado en cuanto a la medición del deseo erótico ya que las conductas que pretende medir son únicamente de origen genital y aporta unos niveles de deseo erótico basados en la cantidad del interés hacia conductas genitales y coitocéntricas.

En la actualidad sigue siendo compleja la medición del deseo erótico además de ser incompleta debido, posiblemente, a la falta de base teórica que confirme el origen del deseo, así como su funcionamiento. El deseo erótico se puede entender como una experiencia emocional subjetiva (Fuertes, 1995) y en este punto, los autores de la presente investigación entienden el componente afectivo del deseo erótico (Salguero et al., 2019) asumiendo que el afecto incluye procesos motivacionales y emocionales (Vohs \& Baumisteir, 2017). Por ello, el objetivo de este estudio se centra en el desarrollo de un instrumento de medida del deseo erótico entendido éste como un proceso afectivo, que 
implica un proceso emocional y motivacional pudiendo llegar a ser regulado y que difiere en su inicio, función y regulación según sean las variables situacionales y personales del sujeto.

\section{Método}

\section{Participantes}

La muestra recogida por muestreo aleatorio está compuesta por 279 sujetos de los cuales un $74 \%$ son mujeres $(\mathrm{n}=207)$ y un $26 \%$ son hombres $(n=72)$. Todos los sujetos que participaron en la investigación tenían pareja. Como criterios de inclusión para la participación en el estudio se usaron los siguientes: ser hombre o mujer mayor de 18 años y tener pareja sentimental en el momento del estudio. Las características sociodemográficas de la muestra se detallan en la Tabla 1. La edad media de la muestra es de 36,2 años siendo el sujeto más joven de 19 años y el mayor de 72 años. El tiempo medio de relación de pareja es de 8,8 años, siendo 47 años y medio el tiempo más largo de relación registrado y un mes el período más corto.

Tabla 1. Características de la Muestra

\begin{tabular}{lc}
\hline Variables & Muestra (n=279) \\
\hline Sexo & \\
Hombre & $72(26 \%)$ \\
Mujer & $207(74 \%)$ \\
Edad Media & 36,2 años \\
Rango & $19($ min) -72 (máx) \\
Tiempo medio de relación & 8,8 años \\
Rango & 1 mes (min) $-47,5$ años (máx) \\
\hline
\end{tabular}

\section{Instrumentos}

Para el presente estudio se les pidió a los sujetos que contestasen el Cuestionario Individual de Deseo Erótico (CIDER) que posteriormente sería analizado. Para ello además, se aplicó el Inventario de Deseo Sexual (SDI) y la Escala de Regulación Emocional (DERS).

\section{Inventario de Deseo Sexual (SDI)}

Este inventario se relaciona con la definición aportada por Spector y colaboradores (1996) acerca del deseo sexual. Se trata de una versión validada al castellano en el año 2006 por Ortega, Zubeidat y Sierra. La versión original es de Spector y colaboradores realizada en 1996 bajo el nombre The Sexual Desire Inventory. La versión original de Spector y colaboradores consta de 13 ítems; los 8 primeros (1-8) hacen referencia al deseo diádico y los 5 últimos (9-13) al deseo en solitario. El instrumento en castellano es un Autoinforme formado por 13 ítems el cual se compone de tres factores. El factor 1 que mide el Deseo Sexual Diádico en pareja y que corresponde a los ítems 1, 2, 3, 6, 7, 8 y 9. Por otro lado el factor 2 que mide el deseo sexual solitario y que corresponde a los ítems $10,11,12$ y 13 y por último el tercer factor que se centra en el deseo sexual diádico hacia una persona atractiva, medido por los ítems 4 y 5. (Moyano et al., 2017).

\section{Escala de Regulación Emocional (DERS)}

El instrumento del que partimos es una adaptación al castellano elaborada por Hervás y Jódar en 2008. La versión original se realiza en el año 2004 por Gratz y Roemer bajo el nombre Difficulties in Emotion Regulation Scale (DERS). La versión adaptada incluye 28 ítems los cuales evalúan diferentes aspectos que pueden causar dificultades incluidos en el proceso de regulación emocional; desatención emocional, interferencia cotidiana, descontrol emocional, confusión emocional y rechazo emocional. El análisis factorial realizado con 5 factores, explicó el $63.5 \%$ de la varianza.

\section{Procedimiento}

En una primera etapa se llevó a cabo la elaboración de una prueba piloto que fue administrada a una pequeña muestra de expertos con la que se pudo evaluar la correcta redacción de los ítems. Posteriormente se evaluó la calidad psicométrica de cada uno de los ítems para después, elaborar el cuestionario. Tras la elaboración de la escala, esta fue suministrada a una muestra incidental de hombres y mujeres con pareja a través de una plataforma de internet. Con ello, y para el posterior análisis, se suministraron además los instrumentos SDI y DERS junto con preguntas relativas a datos sociodemográficos (e.g. sexo, orientación del deseo, tiempo en pareja).

Posteriormente, y con una muestra de 279 personas, se llevó a cabo un análisis factorial exploratorio (AFE) que dio lugar a una escala de 10 ítems formada por tres componentes. Para la realización del estudio se tuvo en cuenta al comité ético el cual no consideró necesario evaluar la investigación por no intervenir directamente con los participantes. 
Tabla 2. Análisis ítem-factor

\begin{tabular}{|c|c|c|c|c|c|}
\hline Factor/Ítem & $M$ & $D T$ & $r^{c}{ }_{i-t}$ & $\alpha-\mathrm{i}$ & $\alpha$ \\
\hline CIDER & 2.63 & .42 & & & .76 \\
\hline Deseo Erótico fuera de la pareja & 2.20 & .28 & & & .79 \\
\hline 3 & 2.26 & .91 & .45 & .74 & \\
\hline 4 & 2.45 & .91 & .54 & .73 & \\
\hline 5 & 1.80 & 1.01 & .38 & .75 & \\
\hline 6 & 2.28 & 10.98 & .57 & .72 & \\
\hline Control de Deseo Erótico & 2.93 & .16 & & & .69 \\
\hline 7 & 3.16 & .84 & .37 & .75 & \\
\hline 8 & 2.93 & .86 & .45 & .74 & \\
\hline 9 & 2.79 & .94 & .35 & .75 & \\
\hline 10 & 2.86 & .97 & .24 & .77 & \\
\hline Deseo Erótico hacia la pareja & 2.88 & .11 & & & 67 \\
\hline 1 & 2.96 & .80 & .60 & .72 & \\
\hline 2 & 2.70 & .95 & .33 & .73 & \\
\hline
\end{tabular}

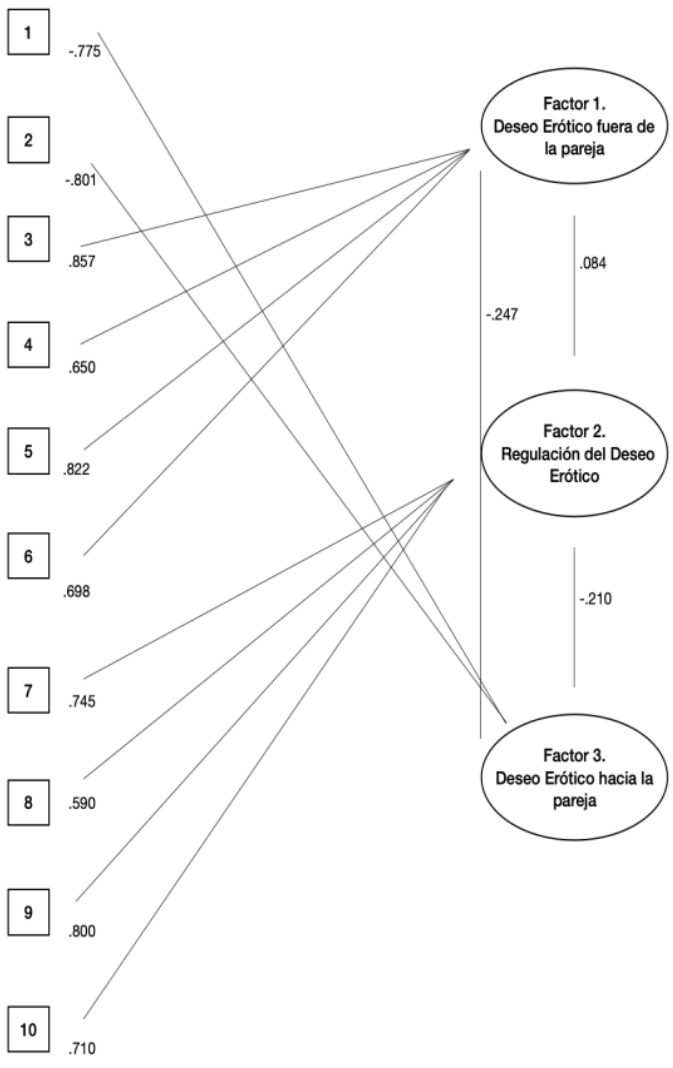

Figura 1. Cargas factoriales estandarizadas del AFE

\section{Resultados}

Para el análisis factorial de los ítems y la escala se llevó a cabo un AFE y se analizaron los diez ítems de forma individual. Para ello se llevó a cabo el método de extracción de componentes principales con el que se analizó la saturación de cada factor.

Con ello se observa la extracción de tres componentes; un primer factor referido al deseo erótico fuera de la pareja, concretamente al deseo autoerótico y al deseo diádico hacia otras personas con las que no hubiera relación sentimental, un segundo factor que hace referencia a la regulación del sujeto sobre su deseo y un último factor referido al deseo diádico hacia la pareja. Se observa que los tres componentes extraídos explican un 63, 6\% de la comunalidad de los datos. En la Figura 1 se muestra el diagrama de Wright (Ferrando \& Anguiano-Carrasco, 2010) en el que se muestran las cargas factoriales estandarizadas en una solución oblicua del modelo de 10 ítems que incluye los tres factores correlados. El factor Deseo erótico hacia la pareja (ítems 1 y 2), el factor Deseo Erótico fuera de la pareja (ítems 3, 4, 5 y 6), el factor Regulación del Deseo Erótico (ítems 7, 8, 9, y 10). En la Figura 1, se detallan las cargas factoriales de los ítems y las correlaciones entre factores.

En la Tabla 2 se detalla el análisis de los ítems del CIDER agrupadas en los diferentes factores. Se presentan las medias, las desviaciones típicas, las correlaciones totales corregidas y el alfa de Cronbach si el ítem es eliminado. En cuanto a la fiabilidad de la escala, la consistencia interna, uno de los criterios fundamentales en la construcción de ítems (Merino, 2020) muestra niveles aceptables para cada factor. El Alfa de Cronbach es de .79 para el factor referido al Deseo Erótico fuera de la pareja, .69 para el segundo factor referido a la Regulación del Deseo Erótico y .67 para el último factor; Deseo Erótico hacia la pareja. Las puntuaciones de los factores de la escala, descritas en las Tablas 3, 4 y 5 mostraron 
Tabla 3. Correlación entre factor Deseo Erótico fuera de la pareja y SDI

\begin{tabular}{|c|c|c|c|c|c|c|}
\hline & Variables & $\begin{array}{l}\text { SDI } \\
\text { (total) }\end{array}$ & $\begin{array}{c}\text { SDI } \\
\text { (diádico) }\end{array}$ & $\begin{array}{c}\text { SDI } \\
\text { (solitario) }\end{array}$ & $\begin{array}{c}\text { SDI } \\
\text { (diádico pareja) }\end{array}$ & $\begin{array}{c}\text { SDI } \\
\text { (diádico pers. } \\
\text { atractiva) }\end{array}$ \\
\hline \multirow{3}{*}{$\begin{array}{l}\text { Deseo Erótico fuera de } \\
\text { la pareja (CIDER) }\end{array}$} & Correlación de Pearson & .58 & .35 & .69 & .19 & .53 \\
\hline & $\mathrm{P}$-value & $<.001$ & $<.001$ & $<.001$ & 0.001 & $<.001$ \\
\hline & $\mathrm{N}$ & 279 & 279 & 279 & 279 & 279 \\
\hline
\end{tabular}

Nota. SDI (total): puntuación total del inventario de deseo sexual. SDI (diádico): ítems referidos al deseo hacia otra persona. SDI (solitario): ítems referidos al deseo autoerótico. SDI (diádico pareja): aquellos ítems referidos únicamente al deseo hacia una pareja sentimental. SDI (diádico pers. atractiva): ítems referidos al deseo erótico diádico hacia una persona atractiva.

Tabla 4. Correlación entre factor Regulación del Deseo Erótico y DERS

\begin{tabular}{llcccccc}
\hline & Variables & DERS & $\begin{array}{c}\text { Atención- } \\
\text { Desatención }\end{array}$ & $\begin{array}{c}\text { Claridad- } \\
\text { Confusión }\end{array}$ & $\begin{array}{c}\text { Aceptación- } \\
\text { Rechazo }\end{array}$ & $\begin{array}{c}\text { Funcionamiento- } \\
\text { Interferencia }\end{array}$ & $\begin{array}{c}\text { Regulación- } \\
\text { Descontrol }\end{array}$ \\
\hline & $\begin{array}{l}\text { Correlación } \\
\text { de Pearson }\end{array}$ & -.31 & -.12 & -.19 & -.20 & -.31 & .31 \\
$\begin{array}{l}\text { Regulación del } \\
\text { Deseo Erótico } \\
\text { (CIDER) }\end{array}$ & P-value & .000 & .218 & .032 & .016 & .000 & .000 \\
& $\mathrm{~N}$ & 132 & 132 & 132 & 132 & 132 & 132 \\
\hline
\end{tabular}

Tabla 5. Correlación entre factor Deseo Erótico hacia la pareja y SDI

\begin{tabular}{|c|c|c|c|c|c|c|}
\hline & Variables & SDI (total) & $\begin{array}{c}\text { SDI } \\
\text { (diádico) }\end{array}$ & $\begin{array}{c}\text { SDI } \\
\text { (solitario) }\end{array}$ & $\begin{array}{l}\text { SDI (diádico } \\
\text { pareja) }\end{array}$ & $\begin{array}{l}\text { SDI (diádico pers. } \\
\text { atractiva) }\end{array}$ \\
\hline \multirow{3}{*}{$\begin{array}{l}\text { Deseo Erótico } \\
\text { hacia la pareja } \\
\text { (CIDER) }\end{array}$} & $\begin{array}{l}\text { Correlación de } \\
\text { Pearson }\end{array}$ & .69 & .74 & .35 & .75 & .17 \\
\hline & P-value & $<.001$ & $<.001$ & $<.001$ & $<.001$ & $<.004$ \\
\hline & $\mathrm{N}$ & 279 & 279 & 279 & 279 & 279 \\
\hline
\end{tabular}

Nota. SDI (total): puntuación total del inventario de deseo sexual. SDI (diádico): ítems referidos al deseo hacia otra persona. SDI (solitario): ítems referidos al deseo autoerótico. SDI (diádico pareja): aquellos ítems referidos únicamente al deseo hacia una pareja sentimental. SDI (diádico pers. atractiva): ítems referidos al deseo erótico diádico hacia una persona atractiva.

altas correlaciones con el SDI para los factores $1 \mathrm{y}$ 3 que hacen referencia a los niveles de deseo erótico y altas correlaciones con el DERS para el factor de Regulación, mostrando así una buena validez convergente en ambos. El factor Regulación del Deseo Erótico muestra correlaciones significativas y negativas con las subescalas de funcionamiento y regulación del DERS, así como una alta correlación con las puntuaciones totales de la escala de regulación.

Tras el análisis factorial, se lleva a cabo un análisis de matriz de correlación con el test de Esfericidad de Barlett además de una medida de adecuación muestral KMO. En la Tabla 6 se detallan los resultados.

Como se observa, el determinante de la matriz de correlaciones arroja un valor cero, lo que indica que el grado de intercorrelación de las variables es muy alto. El test de esfericidad de
Bartlett arroja una significatividad de .000 , por lo que se puede rechazar la hipótesis nula de incorrelación entre variables. El valor KMO arroja un valor superior a 0,80 , por lo que, según este indicador, la matriz de datos resulta apropiada para realizar sobre ella la factorización (Ferrando \& Anguiano-Carrasco, 2010; Lloret-Segura et al., 2014).

Después de llevar a cabo las pruebas de grado de adecuación muestral del AF, se evaluó el ajuste del modelo utilizado mediante el método analítico de rotación oblicua; OBLIMÍN. Se utilizó una solución oblicua dado que es la rotación más congruente con la construcción de instrumentos de variables psicológicas dado que es habitual que los constructos de dicha área se encuentren relacionados en mayor o menor medida (Ledesma et al., 2019). Los autores entienden desde un punto de vista teórico, la relación entre los diferentes 
Tabla 6. Prueba KMO y Barlett

\begin{tabular}{llc}
\hline Medida KMO & & .711 \\
\hline & Aprox. Chi.Cuadrado & 885,893 \\
Prueba de Esfericidad de Barlett & gl. & 45 \\
& Sig. & .000 \\
\hline
\end{tabular}

Nota. KMO (Medida Kaiser-Mayer-Olkin de Adecuación Muestral).

Tabla 7. Matriz de Componente Rotado

\begin{tabular}{lccc}
\hline Ítems & Componente 1 & Componente 2 & Componente 3 \\
\hline 1 & .483 & .190 & -.775 \\
2 & -.025 & .224 & -.801 \\
3 & .857 & .107 & -.138 \\
4 & .650 & .104 & -.617 \\
5 & .822 & .074 & -.090 \\
6 & .698 & .120 & -.590 \\
7 & .064 & .745 & -.267 \\
8 & .160 & .590 & -.485 \\
9 & .091 & .800 & -.129 \\
10 & .039 & .710 & -.013 \\
\hline
\end{tabular}

Nota. Método de rotación Oblimín con normalización Kaiser

factores de la escala que, aún siendo componentes diferenciados, hacen referencia a un mismo constructo. En la Tabla 7 se muestra la Matriz de Componente Rotado.

\section{Discusión}

Los resultados del estudio muestran una escala de medición del deseo y la regulación del mismo compuesta por 3 factores con un total de 10 ítems. El objetivo del estudio fue diseñar un instrumento de medida del deseo erótico que tuviera en cuenta los niveles generales de deseo y la regulación sobre el mismo. De estos resultados, por un lado, se derivan dos factores que harían referencia a los niveles de deseo erótico tanto hacia la pareja como fuera de ella. Como apuntan algunos autores (Spector et.al, 1996) sería un error entender el interés en el comportamiento sexual como sinónimo de conducta sexual real, por tanto, lo que se propuso desde el presente estudio fue la medición de los niveles generales de deseo entendido éste como un proceso afectivo que implica la motivación a generar ciertas conductas eróticas conscientes y voluntarias (Salguero et al., 2019). En relación con la medición de los niveles de deseo erótico, los resultados del estudio ponen de manifiesto la importancia de entender el deseo erótico en pareja como un constructo diferenciado de los niveles de deseo de un sujeto hacia otras personas o en solitario. Como se observa en el AFE, los ítems 1 y 2 conforman un factor en sí mismo destacando, por tanto, que el deseo de un sujeto hacia su pareja debe ser diferenciado de un deseo autoerótico o hacia otras personas.

Así mismo, la marcada relación entre los dos primeros ítems refleja que al preguntar a una persona con pareja sentimental sobre sus niveles generales de deseo erótico, como en el caso del ítem 1, éste responderá en función a la pareja no teniendo en cuenta sus niveles de deseo fuera de ella. Se concluye entonces que el nivel de deseo erótico hacia una pareja, debe medirse y analizarle bajo un prisma diferente a los niveles de deseo hacia otras personas con las que no hay relación sentimental. Esto se relaciona con los datos obtenidos por Moyano, Vallejo-Medina y Sierra (2017), donde tras la validación al castellano del Inventario de Deseo Sexual comentaron la posibilidad de estar frente a tres factores en la escala y no dos, como se apuntó inicialmente. Estos tres factores harían referencia al deseo en 
solitario, al deseo diádico en pareja y al deseo diádico hacia personas atractivas lo que pondría una vez más de manifiesto que el deseo diádico tiene variaciones en su composición y por tanto, en su medición y entendimiento. En palabras de los autores, el deseo erótico en pareja y el deseo hacia personas atractivas advierte claras diferencias puesto que aunque son factores que hacen referencia al deseo diádico, son conceptos diferentes, (Moyano et al. 2017). Además de la medición general del deseo, nuestra escala dio lugar a un tercer factor que haría referencia a la regulación del deseo erótico. Desde la presente investigación se entiende el deseo erótico, como ya afirmó Fuertes (1995), como una experiencia subjetiva de carácter emocional, lo que implica, en nuestra opinión, un componente afectivo del deseo erótico. Entendemos por tanto que ese deseo en cuanto afecto, emoción y motivación, es susceptible de ser regulado (Salguero et al., 2019). En esencia, podemos entender la motivación como una fuerza que actúa dentro del organismo y que tiene como finalidad iniciar o dirigir un comportamiento (Fernández-Abascal, 1995) por ello los ítems de nuestra escala se desarrollaron con la intención de medir la motivación de un sujeto para generar conductas eróticas sin el hecho, por tanto, de que dichas conductas deban generarse. Como apuntan algunos estudios, el comportamiento sexual y el deseo erótico no tienen por qué darse conjuntamente ya que se pueden dar comportamientos sexuales sin deseo erótico previo (Basson, 2000., Beck et al., 1991) o tener altos niveles de deseo sin que se genere una conducta erótica posterior. Desde la Teoría de la Autodeterminación (Ryan \& Deci, 2000) se pone de manifiesto la regulación de la motivación entendida ésta como extrínseca o intrínseca y como activación relacionada, por tanto, con los procesos impulsores de los comportamientos de los sujetos. En el deseo erótico existen diferente variables que estarían influyendo en su inicio y su mantenimiento pudiendo ser éstas de origen interno como las fantasías o de origen externo, como la necesidad de vinculación afectiva. En este sentido y en relación con el deseo erótico femenino, como puso de manifiesto Basson (2000), el deseo de las mujeres tendría que ver con una serie de ganancias a nivel social y afectivo que definirían su inicio y mantenimiento.
Relacionado con esta idea y con la importancia de tener presente las variables situacionales en la medición y entendimiento del deseo, Murnen (1989) indica que frente a una actividad sexual no deseada en las mujeres, las variables situacionales son importantes a la hora de predecir sus reacciones. Desde este estudio se entiende por tanto, que las variables situacionales presentes en el momento del inicio y mantenimiento del deseo erótico podrían ser un indicador importante para comprender la regulación del mismo. Con relación a esto, Kaplan (1978) ya comentó que los síntomas sexuales [...] son producto de la interacción entre el individuo y el medio. Teniendo en cuenta esta idea, en un estudio anterior que tuvo como objetivo analizar la relación entre los niveles de deseo general autoinformado de las personas y una prueba experimental con carga erótica, se concluyó que la situación presente en el momento de la prueba tuvo mayor impacto en los niveles y mantenimiento del deseo erótico que otras variables previas como los niveles autoinformados de dicho constructo (Salguero et al., 2020). El deseo erótico se entendería, por tanto, como un proceso afectivo que tiene como finalidad el desarrollo de conductas eróticas que "atenúen" los determinantes y componentes motivacionales previos, tanto fisiológicos como sociales. En este sentido, se puede entender al sujeto como un ser reactivo que pone en marcha ciertos comportamientos con a la intención de cubrir las demandas y necesidades iniciales (Salguero et al., 2019). Por consiguiente, se podría advertir que el deseo erótico como proceso afectivo no es el único causante de las conductas eróticas que un sujeto pueda llevar a cabo derivándose con ello la idea de que en el desarrollo, inicio y funcionamiento del deseo intervienen múltiples factores, reforzándose así la posibilidad de que no exista una única naturaleza del deseo que pueda ser medible y extrapolable a todos los sujetos sin excepción. Los autores de este trabajo diferencian entre naturaleza del deseo erótico y objeto de deseo. El primero haría referencia a diferentes características que puede presentar el deseo en función de los cambios en los diversos factores que actúan en su activación y desarrollo y el segundo, a los tipos de deseo en función del objeto al que hacen referencia. 
Dicho esto, otros autores ya afirmaron la presencia de diferentes tipos de deseo. En relación con el deseo femenino, Cabello (2010) habló de tres tipos de deseo erótico; un deseo que podría entenderse como fisiológico sin objetivo concreto aparente, un deseo erótico hacia un objeto concreto y un deseo postexcitatorio en el cual la activación de dicho deseo se daría después del inicio de la actividad sexual. Desde un plano cognitivo y como ya se comentó anteriormente, cabría la posibilidad de tener en cuenta la presencia de varios tipos de deseo erótico; solitario, diádico en pareja y diádico hacia personas atractivas (Moyano et al., 2017) lo que destaca la idea derivada de nuestro trabajo de valorar la necesidad del estudio particular del deseo en pareja.

La importancia de tener en cuenta la naturaleza del deseo y de variables situacionales y relacionales que puedan estar interviniendo se reflejaría sin duda, en las implicaciones clínicas. En la actualidad, el abordaje de dificultades en el deseo erótico, y concretamente en casos de deseo sexual hipoactivo, se basarían principalmente en el aumento de fantasías eróticas (Cabello, 2010) que como demuestran algunos estudios estarían muy relacionadas con los niveles de deseo erótico (Zubeidat et al., 2003; Ziegler \& Conley, 2016) y aumento del atractivo en la pareja como ya se puso de manifiesto en el modelo de intervención de McCarthy (1995). Así, uno de los aspectos que creemos fundamental a la hora de intervenir en el deseo erótico desde la clínica, sería entender que el bajo deseo erótico que en ocasiones verbalizan los sujetos y /o parejas no tiene por qué ser siempre motivo o consecuencia de una baja actividad sexual. La nomenclatura de "deseo sexual hipoactivo" no nos parece la más adecuada desde el enfoque aquí presentado manifestando una vez más, que las conductas eróticas no son sinónimo de deseo erótico. En ocasiones puede suceder que no existan inductores externos apropiados para provocar una respuesta (Cabello, 2007). Aunque generalmente ante la existencia de bajos niveles de deseo erótico las conductas eróticas también disminuyen, desde el ámbito clínico debería atenderse a las causas de los bajos niveles de deseo en relación a variables situacionales y relacionales que estarían interfiriendo en el inicio y mantenimiento del mismo y no al abordaje aislado del aumento de las conductas eróticas. Creemos entonces que la intervención debería implicar un trabajo de entendimiento de las variables situacionales y contextuales donde se desarrolla el deseo erótico o la falta del mismo como elementos principales, con la finalidad de que el sujeto aprenda a regular dicho deseo en el contexto concreto donde se inicia o se mantiene y no de forma aislada y general. Esta idea se refuerza con la definición aportada por Cabello (2007) con relación al deseo femenino, quien indica que el DSH podría definirse como una ausencia de pensamientos o fantasías sexuales y de interés en iniciar un encuentro sexual, en presencia de adecuados inductores externos del deseo. Se concluye por tanto, la necesidad de seguir estudiando los procesos involucrados en el inicio y desarrollo del deseo erótico entendiendo éste desde un plano afectivo y desarrollando instrumentos de medida que tengan en cuenta la naturaleza del mismo en función de las variables relacionales, personales y situacionales que puedan estar influyendo.

\section{Referencias}

Basson, R. (2000). The female Sexual Response: A different model. Journal of Sex \& Marital Therapy, 26(1), 51-65. https://doi.org/10.1080/009262300278641

Basson R, Leiblum S, Brotto L, Derogatis L, Fourcroy J, Fugl-Meyer K., Graziottin, A., Heiman, J. R., Laan, E., Meston, C., Shover, L., Lankveld, J., \& Schultz, W. W. (2003). Definitions of women's sexual dysfunction reconsidered: Advocating expansion and revision. Journal of Psychosomatic Obstetrics \& Gynecology, 24(4), 221-9. https://doi.org/10.3109/01674820309074686

Beach, F. A. (1956). Characteristic of masculine "sex drive". In M Jones (ed.), Nebraska Symposium on Motivation (pp. 1-32) Lincoln, University of Nebraska Press.

Beck, G., Bozman, A., \& Qualtrough, B., (1991). The experience of sexual desire: Psychological correlates in a college sample. The Journal of Sex Research, 28(3), 443-456. https://doi.org/10.1080/00224499109551618 
Cabello, F. (2007). Tratamiento del deseo sexual hipoactivo de la mujer. Revista Internacional de Andrología, 5(1), 29-37.

https://doi.org/10.1016/S1698031X(07)74031-1

Cabello, F. (2010). Manual de sexología y terapia sexual. Ed. Síntesis.

Carretero-Dios, H., \& Pérez, C. (2005). Normas para el desarrollo y revisión de estudios instrumentales. International Journal of Clinical and Health Psychology, 5(3), 521551.

Derogatis, L. R., \& Melisaratos, N. (1979). The DSFI: A multidimensional measure of sexual functioning. Journal of Sex \& Marital Therapy, 5(3), 244-281. https://doi.org/10.1080/00926237908403732

Fernández-Abascal, E. (1995). Psicología de la Motivación y la Emoción. En: E. García Fernández-Abascal. (Ed.), Manual de motivación y emoción (pp. 19-52). Centro de Estudios Ramón Areces.

Ferrando, P., \& Anguiano-Carrasco, C. (2010). El análisis factorial como técnica de investigación en psicología. Papeles del Psicólogo 31(1), 18-33.

Freud, S. (1905). Tres ensayos sobre teoría sexual. Omorrortu Editores.

Fuertes, A. (1995). Determinantes relacionales de los problemas de deseo sexual: Pautas para una posible intervención. Anuario de Sexología, I, 27-43.

Fuertes, A. M., \& López, F. S. (1997). Aproximaciones al estudio de la sexualidad. Amarú.

Gil-Verona, J (2009). Neurobiología del Deseo. Anuario de Sexología, 11, 49-53.

Gómez- Zapiain, J. (1995). El deseo sexual y sus trastornos: Aproximación conceptual y etiología. Anuario de Sexología, I, 45-66.

Kaplan, H. S. (1978). La nueva terapia sexual (3º Ed) (Vol.1). Alianza Editorial.

Kaplan, H. S. (1979). Hypoactive sexual desire. Journal of Sex \& Marital Therapy, 3, 3-9. https://doi.org/10.1080/00926237708405343

Kaplan, H. S., \& Guastavino, M. (1982). Trastornos del deseo sexual. Nuevas ideas y técnicas en terapia sexual. Grijalbo.

Ledesma, R., Ferrando, P., \& Tosi, J. (2019). Uso del Análisis Factorial Exploratorio en RIDEP.
Recomendaciones para Autores y Revisores. Revista Iberoamericana de Diagnóstico y Evaluación - e Avaliação Psicológica, 52(3), 173-180.

https://doi.org/10.21865/RIDEP52.3.13

Levine, S. B. (1984). An essay on the nature of sexual desire. Journal of Sex \& Marital Therapy, 10(2), 83-96. https://doi.org/10.1080/00926238408405794

Lloret-Segura, S., Ferreres-Traver, A., HernándezBaeza, A., \& Tomás-Marco, I. (2014). El análisis factorial exploratorio de los ítems: una guía práctica, revisada y actualizada. Anales de Psicología, 30(3), 1151-1169. https://dx.doi.org/10.6018/analesps.30.3.199361

McCarthy, B. W. (1995). Bridges to sexual desire. Journal of Sex Education and Therapy, 21(2), 132-141. https://doi.org/10.1080/01614576.1995.11074 144

Marañón, G. (1925). Patología y higiene de la emoción. Obras Completas (IV). Espasa Calpe S. A.

Masters, W., Johnson, V., \& Kolodny, R. (1994). Heterosexuality. Nueva York: HarperCollins Publishers. (Trad. Grijalbo, 1996).

Merino, C. (2020). Consistencia interna del Eysenck Personality Questionnaire - Revised: Cuando Alfa de Cronbach No Es Suficiente. Revista Iberoamericana de Diagnóstico y Evaluación - e Avaliação Psicológica, 57(4), 191-203. https://doi.org/10.21865/RIDEP57.4.14

Moyano,N., Vallejo-Medina, P., \& Sierra, J. C. (2017).Sexual Desire Inventory. Two or three dimensions?.The Journal of Sex Research, 54(1), 105-116. https://doi.org/10.1080/00224499.2015.1109581

Murnen, S. (1989). Coping with unwanted sexual activity: Normative responses, situational determinants and individual differences. The Journal of Sex Research, 26(1), 85-106. https://doi.org/10.1080/00224498909551493

Nuttin J. (1982). Teoría de la motivación humana. Paidós.

Ortega, V., Zubeidat, I., \& Sierra, J. C. (2006). Further examination of measurement properties of spanish versión of The Sexual Desire Inventory with undergraduates and 
adolescent student. Psychological Reports, 99, 147-165. https://doi.org/10.2466/pr0.99.1

Rose, R., \& Leiblum, S. (1987). Current approaches to the evaluation of sexual desire disorders. Journal of Sex Research, 3(2), 141-162. https://doi.org/10.1080/00224498709551354

Ryan R., \& Deci, E. (2000). Self-determination theory and the facilitation of intrinsic motivation, social development and wellbeing. American Psychologist. 55(1), 1-16. https://doi.org/10.1037110003-066X.55.1.68

Salguero, L., Pérez, M. A., \& Sáez, S. (2019). Una aproximación al deseo erótico como proceso motivacional. Revista Sexología y Sociedad, 25(1), 2-13.

Salguero, L., Pérez, M. A., Sáez, S., \& Gordillo, F. (2020). Impulsivity and the experience of desire in the choice of erotic stimuli. International Journal of Environmental Research and Public Health, 17(14). https://doi.org/10.3390/ijerph17144943

Spector, I., Carey, M., \& Steinberg, L. (1996). The Sexual Desire Inventory: Development, factor structure and evidence of reliability. Journal of Sex \& Marital Therapy, 22 (3), 175-190. https://doi.org/10.1080/00926239608414655

Vohs K., \& Baumisteir R. (2017). Handbook of self-regulation: Research, theory and applications. 3rd ed. Gilford Press, p. 3-23.

Ziegler, A., \& Conley, T. (2016). The importance and meaning of sexual fantasies in intimate relationships. In K. Aumer (ed.), The Psychology of Love and Hate in Intimate Relationships (1st ed., pp. 29-45). Springer.

Zubeidat, I., Ortega, V., Villar, C., \& Sierra, J. C. (2003). Un estudio sobre la implicación de las actitudes y fantasías sexuales en el deseo sexual de los adolescentes. Cuadernos de Medicina Psicosomática y Psiquiatría de Enlace, (67-68), 71-78. 


\section{Anexo I}

A continuación usted responderá unas preguntas acerca de su deseo erótico. Entendiendo Deseo Erótico como las ganas o motivación por generar situaciones eróticas.

Por favor, responda en relación a la escala que se le presenta a continuación:

1-NADA 2-POCO 3-BASTANTE 4-MUCHO

\begin{tabular}{|c|c|c|c|c|}
\hline 1. ¿Cómo califica su nivel de deseo erótico, en general? & 1 & 2 & 3 & 4 \\
\hline $\begin{array}{l}\text { 2. ¿Cómo calificaría el nivel de deseo erótico hacia su pareja en las últimas } \\
\text { semanas? }\end{array}$ & 1 & 2 & 3 & 4 \\
\hline 3. En general, ¿cómo califica su nivel de deseo erótico hacia otras personas? & 1 & 2 & 3 & 4 \\
\hline 4. En general, ¿cómo calificaría su nivel de deseo erótico en solitario? & 1 & 2 & 3 & 4 \\
\hline $\begin{array}{l}\text { 5. En las últimas semanas, ¿qué ganas ha tenido de generar situaciones } \\
\text { eróticas con personas con las que no tiene una relación sentimental? }\end{array}$ & 1 & 2 & 3 & 4 \\
\hline $\begin{array}{l}\text { 6. En las últimas semanas, ¿qué ganas ha tenido de mantener conductas } \\
\text { eróticas consigo mismo? }\end{array}$ & 1 & 2 & 3 & 4 \\
\hline 7. ¿Cuánto cree que es el nivel de control que tiene sobre su deseo erótico? & 1 & 2 & 3 & 4 \\
\hline $\begin{array}{l}\text { 8. Piense en situaciones que han activado su deseo erótico en las últimas } \\
\text { semanas. ¿Ha sentido control sobre su deseo pudiendo aumentarlo? }\end{array}$ & 1 & 2 & 3 & 4 \\
\hline $\begin{array}{l}\text { 9. Piense en situaciones que han activado su deseo erótico en las últimas } \\
\text { semanas. ¿Ha sentido control sobre su deseo pudiendo disminuirlo? }\end{array}$ & 1 & 2 & 3 & 4 \\
\hline $\begin{array}{l}\text { 10.Cuando ha percibido que una persona atractiva interacciona con usted } \\
\text { con tono erótico, ¿en qué medida ha regulado su deseo? }\end{array}$ & 1 & 2 & 3 & 4 \\
\hline
\end{tabular}

